\title{
High MMP-26 expression in glioma is correlated with poor clinical outcome of patients
}

\author{
JIAN-GUI GUO ${ }^{1}$, CHENG-CHENG GUO ${ }^{2-4}$, ZHEN-QIANG HE ${ }^{2-4}$, XIU-YU CAI ${ }^{2,3,5}$ and YONG-GAO MOU ${ }^{2-4}$ \\ ${ }^{1}$ Department of Radiation Oncology, The First People's Hospital of Foshan, Foshan, Guandong 528000; \\ ${ }^{2}$ State Key Laboratory of Oncology in South China, Cancer Center, Sun Yat-Sen University; \\ ${ }^{3}$ Collaborative Innovation Center for Cancer Medicine; ${ }^{4}$ Department of Neurosurgery, \\ Cancer Center, Sun Yat-Sen University; ${ }^{5}$ Department of VIP Region, Cancer Center, \\ Sun Yat-Sen University, Guangzhou, Guandong 510060, P.R. China
}

Received December 11, 2017; Accepted May 22, 2018

DOI: $10.3892 / \mathrm{ol} .2018 .8880$

\begin{abstract}
To date the management of glioma remains a great challenge in cancer therapy worldwide. The identification of novel diagnostic and therapeutic methods is required. Although there is data indicating that matrix metalloproteinase (MMP)-26 serves an important role in many human cancer types, its clinical significance in glioma remains uncertain. The present study aimed to evaluate MMP-26 expression in human astrocytic glioma specimens, and investigate its role and significance in the progression of astrocytic glioma. Immunohistochemistry was performed to assess MMP-26 expression in astrocytic glioma tissues. The levels of MMP-26 expression and its relevance to the clinicopathological features and prognostic factors in patients with astrocytic glioma patients were then investigated. The results demonstrated that MMP-26 expression was significantly assocaited with the World Health Organization grade $(\mathrm{P}<0.05)$. Additionally, it was identified that MMP-26 expression was an effective predictor of the overall survival of patients with astrocytic glioma $(\mathrm{P}<0.05)$. Analyses of univariate and multivariate Cox regression confirmed that MMP-26 expression was an independent factor for evaluating the prognosis of astrocytic glioma patients $(\mathrm{P}<0.05)$. The current results support that MMP-26 may be a novel indicator of diagnosis and an independent factor for evaluating prognosis in patients with glioma.
\end{abstract}

Correspondence to: Dr Yong-Gao Mou, Department of Neurosurgery, Cancer Center, Sun Yat-Sen University, 651 Dongfeng East Road, Guangzhou, Guandong 510060, P.R. China

E-mail: mouyg@sysucc.org.cn

Dr Xiu-Yu Cai, Department of VIP Region, Cancer Center, Sun Yat-Sen University, 651 Dongfeng East Road, Guangzhou, Guandong 510060, P.R. China

E-mail: caixy@sysucc.org.cn

Key words: MMP-26, astrocytic glioma, immunohistochemistry, prognosis

\section{Introduction}

Astrocytic glioma represents the most prevalent form of malignant tumor to occur in the primary central nervous system (CNS) of adults (1). The 5-year survival rate in glioma patients remains lower than that for other cancers in the United States of America (2). Despite the progress that has been made in conventional therapies including neurosurgery, radiotherapy and chemotherapy, the prognosis of patients with glioma remains poor $(3,4)$. Due to this markedly poor prognosis and lack of effective therapeutic options, a greater understanding of the molecular properties of glioma to develop effective diagnostic and therapeutic strategies is urgently required.

The matrix metalloproteinase (MMP) family is a group of zinc-dependent endopeptidases that share similar structure and serve as main regulators in the process of tumorigenesis (5). Previous studies have indicated MMPs are important in a number of physiological processes, including in the degradation of basal epithelial membranes and various extracellular matrix (ECM) components, in apoptosis, angiogenesis and inflammation, and also in cell growth, migration and differentiation (5). Research has also demonstrated that the degradation of the ECM served a crucial role in the process of glioma cell invasion into surrounding brain tissue (6). Furthermore, MMP-13 was indicated to be associated with the World Health Organization (WHO) grade and as a potential prognostic marker in glioma (7). In addition, numerous other MMP family members, including MMP-14, MMP-19 and MMP-28 have been confirmed to be correlated with the WHO grade and have prognostic value in glioma $(8,9)$. Aberrant expression of the MMP family members has been closely associated with the pathological process of glioma, and therefore MMPs have potential as diagnostic molecular biomarkers in human glioma.

Matrix metalloproteinase-26 (MMP-26) belongs to a large ECM protease family $(10,11)$. MMP-26 is a human-specific protease that was first cloned from a human endometrial tumor cDNA library in $2000(10,11)$. The lack of a hinge region distinguished it from other MMPs (12). MMP-26 has the capacity to effectively degrade various components of the ECM, including fibronectin, gelatins, vitronectin and 
fibrinogen (13). In particular, published research has demonstrated that MMP-26 served a critical role in the invasion and angiogenesis of glioma cells (14). However, the clinical and prognostic significance of MMP-26 in glioma need to be investigated further.

In the present study, the expression of MMP-26 in human astrocytic glioma specimens was detected, to investigate its role and significance in the progression of glioma.

\section{Materials and methods}

Clinical data and tissue samples. Clinical paraffin-embedded astrocytic glioma tissue samples were obtained from 120 patients who had received tumor excision surgery between January 2000 and December 2008. All the slides were re-evaluated according to the 2007 WHO classification of tumors of the central nervous system criteria (4th edition) by two pathologists, with differences resolved by careful discussion. None of the patients had received anti-cancer treatments prior to operation. Overall survival (OS) was based on patient operative time as a starting point, to patient fatality, loss at follow-up or the last follow-up. The follow-up information was updated every three months and a follow-up of at least five years was performed. The complete follow-up information was obtained in the current research. The Sun Yat-Sen University Cancer Center ethics committee reviewed and approved the current study and each patient signed written informed consent at the Cancer Center of Sun Yat-Sen University.

Immunohistochemical staining. Immunohistochemistry staining was performed on $5 \mu \mathrm{m}$ sections of the paraffin-embedded astrocytic glioma tissue samples using an SPlink Detection kits (cat. no. SP-9000; ZSGB-Bio, Beijing, China) based on the protocols of the manufacturer. In brief, the sections were first transferred to adhesive slides and dried at $60^{\circ} \mathrm{C}$ for at least $30 \mathrm{~min}$. Xylene was then used to deparaffinize the sections and rehydrate them with a decreasing alcohol gradient and double-distilled water. The heat-induced antigen retrieval was performed in citrate buffer by boiling for $10 \mathrm{~min}$. Subsequently, the sections were treated with $3 \%$ hydrogen peroxide $\left(\mathrm{H}_{2} \mathrm{O}_{2}\right)$ to remove endogenous peroxides. Then, the sections were incubated with MMP-26 antibody (cat. no. ab81285; dilution 1:50; Abcam, Cambridge, MA, USA). Human colorectal cancer tissues were used as a positive control. Phosphate buffer solution replaced MMP-26 antibody, which served as a negative control.

Evaluation of MMP-26 staining. The immunohistochemical staining results were analyzed according to a previously described method (15). The percentage of MMP-26-positive cells was scored as 0 for $<5 \%, 1$ for $5-<25 \%, 2$ for $25-50 \%$ and 3 for $>50 \%$ cell staining. The intensity of staining was scored as 0 for no staining, 1 for pallide-flavens, 2 for yellow and 3 for brown staining. The MMP-26 immunohistochemical score was defined by multiplying the positive cells percentage and staining intensity scores. Samples were then divided into two groups according to the score: Scores $\leq 4$ defined a low MMP-26 expression group while scores $>4$ defined a high
MMP-26 expression group. The immunohistochemical scoring was conducted by two independent pathologists blinded to the information on the patient's clinical characteristics.

Statistical analysis. The data were analyzed using SPSS software (version 22.0; IBM Corp., Armonk, NY, USA). The $\chi^{2}$ test was used to analyze associations between MMP-26 expression and clinicopathological features. The Kaplan-Meier method was used to estimate survival from survival curves, and the log-rank test was used to calculate the difference between survival curves. Statistical significance was defined at $\mathrm{P}<0.05$.

\section{Results}

Study population. Table I presents the clinicopathological features of the 120 patients investigated in the current study. Among them, 90 patients $(75.0 \%)$ succumbed to mortality before the end of the follow-up period. The patient's median age was 42 (range, 2-75) years. A total of 89 patients (74.2\%) had received total tumor resection. Among the patients, 40 cases $(33.3 \%)$ were classified as grade II, 31 cases $(25.9 \%)$ as grade III and 49 cases $(40.8 \%)$ as grade IV, based on the WHO grading standards. The median follow-up period was 51.8 (range, 2.0-156.0) months.

Immunohistochemical characteristics. In the immunohistochemistry analysis, low MMP-26 protein expression was determined in 89 of the $120(74.2 \%)$ paraffin-embedded astrocytic glioma tissues, and high MMP-26 protein expression was detected in the remaining cases (31 of 120, 25.8\%). Immunoreactivity of MMP-26 was mainly detected in the cytoplasm (Fig. 1).

Associations between MMP-26 expression and clinical characteristics. The associations between MMP-26 expression and the clinical features of the patients with astrocytic glioma are presented in Table II. The results demonstrated that MMP-26 expression was significantly related to tumor WHO grade $(\mathrm{P}=0.006)$, but did not reveal significant differences associated with patient age, sex distribution, Karnofsky performance status score, extent of resection or tumor location in the astrocytic glioma patients $(\mathrm{P}>0.05)$.

Prognostic value of MMP-26 expression in astrocytic glioma. The prognostic value of MMP-26 expression was analyzed by comparing OS according to MMP-26 expression levels. The data demonstrated that the difference in the OS rate between the high and low expression groups was statistically significant (Fig. 2; P<0.001) according to the Kaplan-Meier survival analysis. Separate analysis of the difference of the OS rate between the MMP-26 expression groups according to WHO grade also revealed a statistically significant difference (Fig. 3; P<0.05). Univariate and multivariate survival analyses adjusting for all of the available clinicopathological parameters of patients are presented in Table III. The analyses of the univariate and multivariate Cox regression indicated that age, WHO grade and MMP-26 expression were independent factors for evaluating the prognosis of patients with astrocytic glioma (Table III; $\mathrm{P}<0.05$ ). 
Table I. Clinical characteristics of the 120 glioma patients.

\begin{tabular}{lr}
\hline Characteristic & Number $(\%)$ \\
\hline Age (years) & \\
Median & 42 \\
Range & $2-75$ \\
Sex & \\
Male & $70(58.3)$ \\
Female & $50(41.7)$ \\
WHO grade & \\
II & $40(33.3)$ \\
III & $31(25.9)$ \\
IV & $49(40.8)$ \\
KPS & \\
$\geq 70$ & $113(94.2)$ \\
<70 & $7(5.8)$ \\
Extent of resection & \\
Total & $89(74.2)$ \\
Subtotal & $31(25.8)$ \\
Location & \\
Supratentorial & $111(92.5)$ \\
Infratentorial & $9(7.5)$ \\
Mortality & $30(25.0)$ \\
No & $90(75.0)$ \\
Yes & \\
\hline
\end{tabular}

KPS, Karnofsky performance status; WHO, World Health Organization.

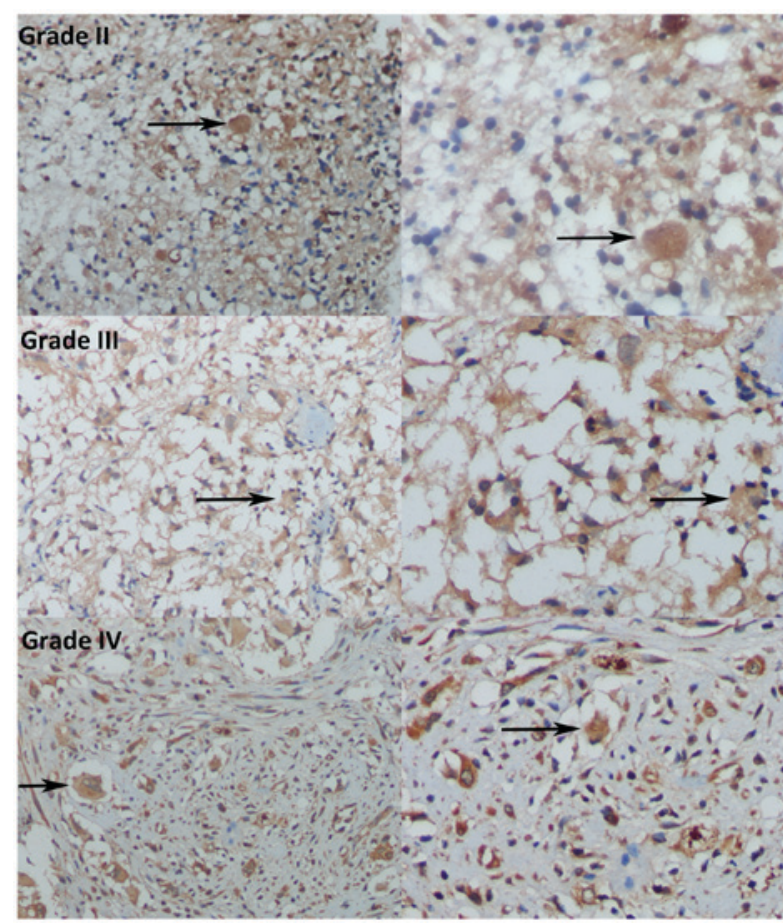

Figure 1. Immunohistochemical analysis of MMP-26 expression in glioma human brain specimens of varying World Health Organization grades. Grade II, grade III, and grade IV glioma specimens (magnification, left, $\mathrm{x} 200$; right, $\mathrm{x} 400$ ) immunohistochemically stained for MMP-26 are shown. Blue staining indicates the nuclei of glioma cells; brown staining indicates MMP-26 expression in the cytoplasm, with the black arrows indicating regions of strong staining. MMP-26, matrix metalloproteinase-26.
Table II. Associations of MMP-26 expression with the clinicopathological features of glioma patients.

\begin{tabular}{|c|c|c|c|c|}
\hline \multirow[b]{2}{*}{ Variable } & \multicolumn{2}{|c|}{$\begin{array}{l}\text { MMP-26 } \\
\text { expression }\end{array}$} & \multirow[b]{2}{*}{ P-value } & \multirow[b]{2}{*}{$\chi^{2}$ value } \\
\hline & Low & High & & \\
\hline All cases & 89 & 31 & & \\
\hline \multicolumn{5}{|l|}{ Sex } \\
\hline Male & 51 & 19 & & \\
\hline Female & 38 & 12 & 0.698 & 0.150 \\
\hline \multicolumn{5}{|l|}{ Age (years) } \\
\hline$\geq 40$ & 45 & 13 & & \\
\hline$<40$ & 44 & 18 & 0.408 & 0.685 \\
\hline \multicolumn{5}{|l|}{ KPS } \\
\hline$\geq 70$ & 83 & 30 & & \\
\hline$<70$ & 6 & 1 & 0.784 & 0.582 \\
\hline \multicolumn{5}{|c|}{ Extent of resection } \\
\hline Total & 66 & 23 & & \\
\hline Subtotal & 23 & 8 & 0.997 & $<0.001$ \\
\hline \multicolumn{5}{|l|}{ Location } \\
\hline Supratentorial & 82 & 29 & & \\
\hline Infratentorial & 7 & 2 & 0.794 & 0.068 \\
\hline \multicolumn{5}{|l|}{ WHO grade } \\
\hline II & 35 & 5 & & \\
\hline III & 25 & 6 & & \\
\hline IV & 29 & 20 & $0.006^{\mathrm{a}}$ & 10.132 \\
\hline
\end{tabular}

${ }^{\mathrm{a}}<0.05$ was considered significant. KPS, Karnofsky performance status; WHO, World Health Organization; MMP, matrix metalloproteinase.

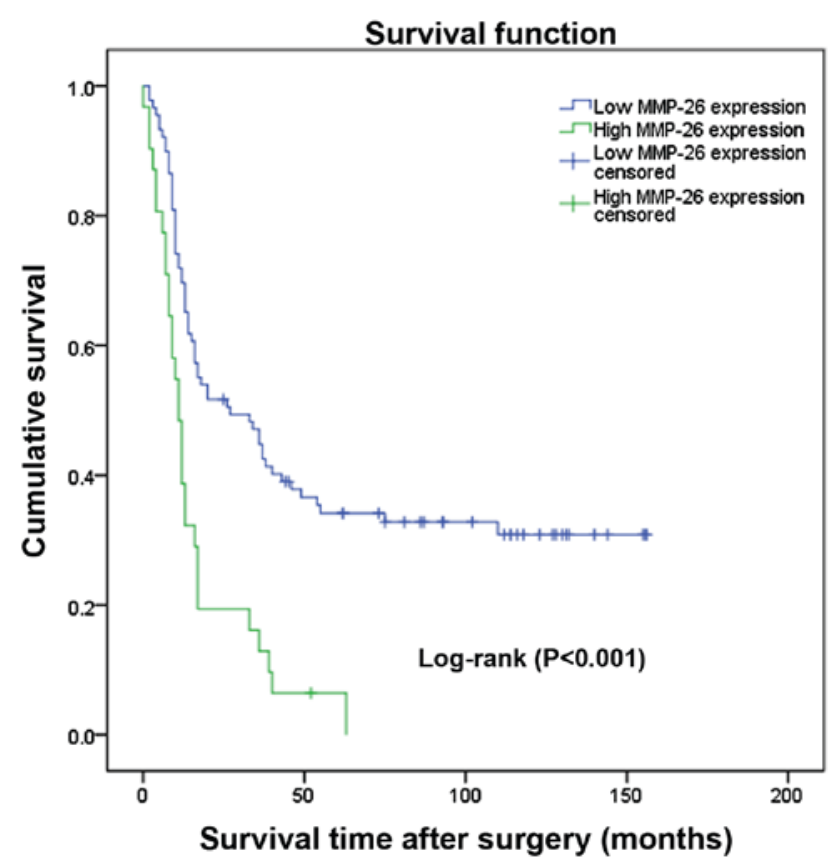

Figure 2. Cumulative survival curves of the glioma patients following surgery according to MMP-26 expression levels $(\mathrm{n}=120)(\mathrm{P}<0.001)$. MMP-26, matrix metalloproteinase- 26 . 
Table III. Univariate and Multivariate Cox regression analyses of patient survival.

\begin{tabular}{|c|c|c|c|c|c|c|}
\hline \multirow[b]{2}{*}{ Variable } & \multicolumn{3}{|c|}{ Univariate } & \multicolumn{3}{|c|}{ Multivariate } \\
\hline & HR & $95 \% \mathrm{CI}$ & P-value & HR & $95 \% \mathrm{CI}$ & P-value \\
\hline Sex (male vs. female) & 0.709 & $0.464-1.085$ & 0.113 & 0.821 & $0.277-1.438$ & 0.383 \\
\hline Age $(\geq 40$ years vs. $<40$ years $)$ & 2.506 & $1.623-3.871$ & $<0.001^{\mathrm{a}}$ & 2.170 & $0.467-3.909$ & $0.001^{\mathrm{a}}$ \\
\hline KPS ( $\geq 70$ vs. $<70)$ & 0.162 & $0.787-4.181$ & 0.162 & 0.950 & $0.023-2.751$ & 0.909 \\
\hline Extent of resection (total vs. subtotal) & 1.389 & $0.884-2.182$ & 0.154 & 1.552 & $0.345-3.320$ & 0.071 \\
\hline Tumor location (supratentorial vs. infratentorial) & 0.295 & $0.093-0.935$ & 0.038 & 0.697 & $0.991-12.140$ & 0.557 \\
\hline WHO grade (T2/T3/T4) & 1.828 & $1.424-2.346$ & $<0.001^{\mathrm{a}}$ & 1.573 & $1.565-6.090$ & $0.001^{\mathrm{a}}$ \\
\hline MMP-26 (low vs. high) & 2.600 & $1.657-4.080$ & $<0.001^{\mathrm{a}}$ & 1.865 & $0.352-2.360$ & $0.009^{\mathrm{a}}$ \\
\hline
\end{tabular}

${ }^{\mathrm{a}} \mathrm{P}<0.05$ was considered significant. KPS, Karnofsky performance status; WHO, World Health Organization; HR, hazard ratio; CI, confidence interval; MMP, matrix metalloproteinase.

A

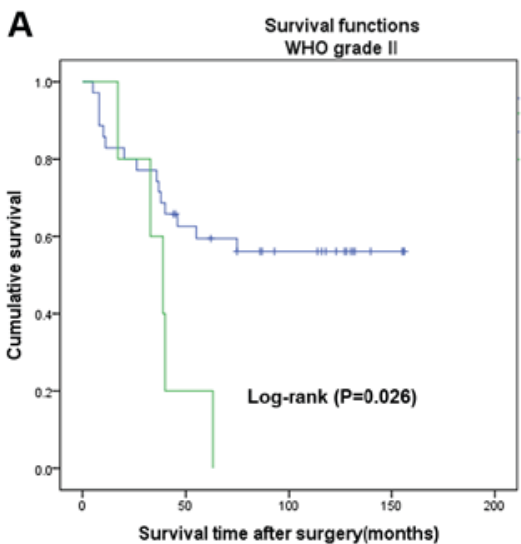

B

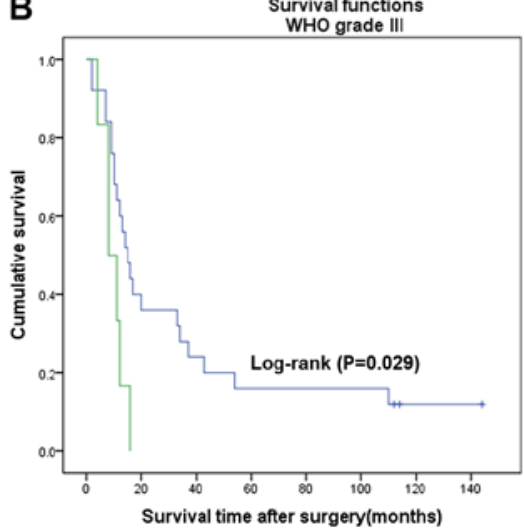

C Survival functions

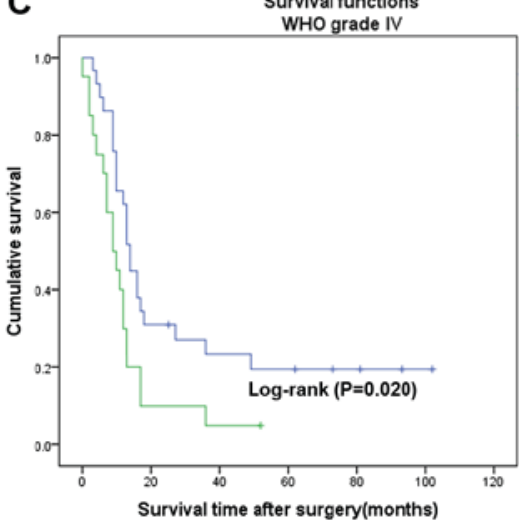

Figure 3. Cumulative survival curves of glioma patients following surgery according to MMP-26 expression stratified by WHO grade. (A) Survival curves for the 40 patients with grade II $(\mathrm{P}=0.026)$. (B) Survival curves for the 31 patients with grade III ( $\mathrm{P}=0.029)$. (C) Survival curves for the 49 patients with grade IV $(\mathrm{P}=0.020)$. WHO, World Health Organization. MMP-26, matrix metalloproteinase-26.

\section{Discussion}

Primary CNS tumors represented $1.38 \%$ of all malignant tumors and accounted for $2.60 \%$ of all malignant tumor-associated mortalities in the United States in 2015 (2). Astrocytic glioma, which is the most prevalent and lethal form of primary tumor of the CNS, has a highly unfavorable prognosis. Statistics indicate that the median OS time of patients with glioblastoma (GBM) is no more than fifteen months, and that the 5-year survival rate is less than $10 \%$ (3). Despite the possibility for early detection and advancements in various therapeutic methods, glioma continues to pose a serious health threat to human health (16). It is well known that the prognosis of glioma has a strong relationship with local recurrence and progression. With the development of molecular biology, it is important to identify sensitive biomarkers that can not only identify early local recurrence and progression, but which also have the versatility to be used as suitable targets for therapy.

In the present study, research was conducted on MMP-26 expression in astrocytic glioma and its clinical significance. MMP-26 expression was evaluated in 120 cases of paraffin-embedded astrocytic glioma tissue samples, and its correlation with the clinicopathological parameters of patients was assessed. According to the results, it was identified that high MMP-26 expression was more likely to occur in advanced grade astrocytic glioma, and furthermore, it was confirmed that MMP-26 expression was correlated with WHO grade. However, no significant relationships with other clinicopathological parameters were identified. Previous studies have indicated the possible participation of MMP-26 in tumor invasion and metastasis (17-19). Hu et al identified that the expression of MMP-26 was correlated with cancer invasion and metastasis and served an important role in tumor progression in colorectal cancer (15). Yamamoto et al confirmed that MMP-26 expression was significantly correlated with cancer invasion, metastasis and recurrence in esophageal squamous cell carcinoma (20). The present data also implied that MMP-26 may serve a vital role in glioma progression. Thus, MMP-26 may play a similar role in the invasion and progression of glioma to that observed in many other cancers.

In addition, the present results demonstrated that high MMP-26 expression in glioma was independently associated with poor rates of OS in patients following tumor resection. Kaplan-Meier analysis indicated that patients with high MMP-26 expression tended to have a lower OS rate compared with in patients with low MMP-26 expression, suggesting that 
high MMP-26 expression levels indicated poor prognosis in astrocytic glioma patients. In addition, univariate and multivariate analyses adjusting all of the available clinicopathological parameters of patients, demonstrated that MMP-26 was an independent factor associated with the OS time of the astrocytic glioma patients, in addition to the generally established clinical predictors of age and WHO grade. Thus, MMP-26 may be regarded as a possible biomarker for predicting the OS time of astrocytic glioma patients, and also as an independent prognostic index for patients with astrocytic glioma. As the number of the patients we have enrolled is small and we have only detected the MMP-26 protein expression by immunohistochemistry, further studies referring to the MMP-26 expression in mRNA and gene level should be conducted to confirm the prognostic significance of MMP-26 expression in astrocytic glioma. At present, the mechanism of MMP-26 in enhancing tumor invasion and predicting the OS of glioma patients is not clear. A previous study demonstrated that MMP-26 served a key role in the invasion process of glioma cells by impacting on ECM components in vitro (14). Additionally, MMP-26 has been reported to promote glioma cell invasion into the tissues surrounding the tumor (14). Furthermore, proMMP-9 could be activated by MMP-26, which is dependent upon cleavage of the Ala93-Met94 site in the proenzyme $(20,21)$. TIMP4 may also play an important role in the interactions with MMP-26 to promote the cancer carcinogenesis and progression (22-24). However, the underlying mechanisms should be clarified in greater detail.

In conclusion, the present study confirmed that MMP-26 expression was significantly associated with WHO grade, and that MMP-26 was an independent predictor of OS in glioma patients. The current findings indicated that MMP-26 may serve as a novel indicator for the diagnosis and in evaluating the prognosis of patients with glioma. Since the number of the patients we studied is small and we have only detected the MMP-26 protein expression by immunohistochemistry, further studies should be taken to confirm the prognostic significance of MMP-26 expression in glioma.

\section{Acknowledgements}

Not applicable.

\section{Funding}

This study was supported by the Science and Technology Planning Project of Guangdong Province, China (grant nos. Z012B031800382 and 2014A020212098) and National Natural Science Foundation of China (grant no. 81401908).

\section{Availability of data and materials}

The datasets used and/or analyzed during the current study are available from the corresponding author on reasonable request

\section{Authors' contributions}

YGM and XYC were involved in conception and design of the study; YGM provided administrative support; JGG, CCG and $\mathrm{ZQH}$ were involved in provision of study materials and patients;
JGG and CCG were involved in collection and assembly of data; data analysis and interpretation, and manuscript writing were performed by JGG, XYC and ZQH. All authors approved the final version of the manuscript.

\section{Ethics approval and consent to participate}

The Sun Yat-Sen University Cancer Center ethics committee reviewed and approved the current study and each patient signed written informed consent at the Cancer Center of Sun Yat-Sen University.

\section{Consent for publication}

Not applicable.

\section{Competing interests}

The authors declare no conflicts of interests.

\section{References}

1. Ostrom QT, Gittleman H, Fulop J, Liu M, Blanda R, Kromer C, Wolinsky Y, Kruchko C and Barnholtz-Sloan JS: CBTRUS statistical report: Primary brain and central nervous system tumors diagnosed in the united states in 2008-2012. Neuro Oncol 17 (Suppl 4): ivl-iv62, 2015.

2. Siegel RL, Miller KD and Jemal A: Cancer statistics, 2015. CA Cancer J Clin 65: 5-29, 2015.

3. Stupp R, Hegi ME, Mason WP, van den Bent MJ, Taphoorn MJ, Janzer RC, Ludwin SK, Allgeier A, Fisher B, Belanger K, et al: Effects of radiotherapy with concomitant and adjuvant temozolomide versus radiotherapy alone on survival in glioblastoma in a randomised phase III study: 5-year analysis of the EORTC-NCIC trial. Lancet Oncol 10: 459-466, 2009.

4. Stupp R, Mason WP, van den Bent MJ, Weller M, Fisher B, Taphoorn MJ, Belanger K, Brandes AA, Marosi C, Bogdahn U, et al: Radiotherapy plus concomitant and adjuvant temozolomide for glioblastoma. N Engl J Med 352: 987-996, 2005.

5. Malemud CJ: Matrix metalloproteinases (MMPs) in health and disease: An overview. Front Biosci 11: 1696-1701, 2006.

6. Liu L, Wu J, Ying Z, Chen B, Han A, Liang Y, Song L, Yuan J, $\mathrm{Li} \mathrm{J}$ and $\mathrm{Li} \mathrm{M}$ : Astrocyte elevated gene-1 upregulates matrix metalloproteinase-9 and induces human glioma invasion. Cancer Res 70: 3750-3759, 2010.

7. Wang J, Li Y, Li C, Yu K and Wang Q: Increased expression of matrix metalloproteinase-13 in glioma is associated with poor overall survival of patients. Med Oncol 29: 2432-2437, 2012.

8. Wang L, Yuan J, Tu Y, Mao X, He S, Fu G, Zong J and Zhang Y: Co-expression of MMP-14 and MMP-19 predicts poor survival in human glioma. Clin Transl Oncol 15: 139-145, 2013.

9. Wang X, Zhang K, Chen X, Zhao C and Sun Z: Epilysin is overexpressed in glioblastoma and related to clinical outcome of patients. Med Oncol 32: 363, 2015.

10. Park HI, Ni J, Gerkema FE, Liu D, Belozerov VE and Sang QX: Identification and characterization of human endometase (matrix metalloproteinase-26) from endometrial tumor. J Biol Chem 275: 20540-20544, 2000

11. Uria JA and López-Otin C: Matrilysin-2, a new matrix metalloproteinase expressed in human tumors and showing the minimal domain organization required for secretion, latency, and activity. Cancer Res 60: 4745-4751, 2000.

12. de Coignac AB, Elson G, Delneste Y, Magistrelli G, Jeannin P, Aubry JP, Berthier O, Schmitt D, Bonnefoy JY and Gauchat JF: Cloning of MMP-26. A novel matrilysin-like proteinase. Eur J Biochem 267: 3323-3329, 2000.

13. Marchenko GN, Ratnikov BI, Rozanov DV, Godzik A, Deryugina EI and Strongin AY: Characterization of matrix metalloproteinase-26, a novel metalloproteinase widely expressed in cancer cells of epithelial origin. Biochem J 356: 705-718, 2001.

14. Deng Y, Li W, Li Y, Yang H, Xu H, Liang S, Zhang L and Li Y: Expression of Matrix Metalloproteinase-26 promotes human glioma U251 cell invasion in vitro and in vivo. Oncol Rep 23: 69-78, 2010. 
15. Hu Q, Yan C, Xu C, Yan H, Qing L, Pu Y, He Z and Li X: Matrilysin-2 expression in colorectal cancer is associated with overall survival of patients. Tumour Biol 35: 3569-3574, 2014.

16. Chang SM, Butowski NA, Sneed PK and Garner IV: Standard treatment and experimental targeted drug therapy for recurrent glioblastoma multiforme. Neurosurg Focus 20: E4, 2006.

17. Bister V, Skoog T, Virolainen S, Kiviluoto T, Puolakkainen P and Saarialho-Kere U: Increased expression of matrix metalloproteinases-21 and -26 and TIMP-4 in pancreatic adenocarcinoma. Mod Pathol 20: 1128-1140, 2007.

18. Li L, Mei TH, Zhou XD and Zhang XG: Expression and clinical significance of matrix metalloproteinase (MMP)-26 protein in non-small cell lung cancer. Ai Zhengr 28: 60-63, 2009.

19. Yang H, Wang Y, Li Y, Zhang L, Deng Y, Qi D, Li Y and Li W: Roles of matrix metalloproteinase-26 in the growth, invasion and angiogenesis of breast cancer. Oncol Lett 4: 832-836, 2012.

20. Yamamoto H, Vinitketkumnuen A, Adachi Y, Taniguchi $H$, Hirata T, Miyamoto N, Nosho K, Imsumran A, Fujita M, Hosokawa M, et al: Association of matrilysin-2 (MMP-26) expression with tumor progression and activation of MMP-9 in esophageal squamous cell carcinoma. Carcinogenesis 25: 2353-2360, 2004.
21. Zhao YG, Xiao AZ, Newcomer RG, Park HI, Kang T, Chung LW, Swanson MG, Zhau HE, Kurhanewicz J and Sang QX: Activation of pro-gelatinase B by endometase/matrilysin-2 promotes invasion of human prostate cancer cells. J Biol Chem 278: 15056-15064, 2003.

22. Zhang J, Cao YJ, Zhao YG, Sang QX and Duan EK: Expression of matrix metalloproteinase-26 and tissue inhibitor of metalloproteinase-4 in human normal cytotrophoblast cells and a choriocarcinoma cell line, JEG-3. Mol Hum Reprod 8: 659-666, 2002.

23. Zhao YQ, Wang QY, Zhai M, Xu J, Chen XQ, Liu WL, Zhang M, Song SJ, Wang JM, Meng FY and Shan YD: A multi-center clinical trial of recombinant human thrombopoietin in chronic refractory idiopathic thrombocytopenic purpura. Zhonghua Nei Ke Za Zhi 43: 608-610, 2004 (In Chinese).

24. Lee S, Desai KK, Iczkowski KA, Newcomer RG, Wu KJ, Zhao YG, Tan WW, Roycik MD and Sang QX: Coordinated peak expression of MMP-26 and TIMP-4 in preinvasive human prostate tumor. Cell Res 16: 750-758, 2006.

This work is licensed under a Creative Commons Attribution-NonCommercial-NoDerivatives 4.0 International (CC BY-NC-ND 4.0) License. 